\title{
Experimental Observation of Topologically Protected Helical Edge Modes in Patterned Elastic Plates
}

\author{
M. Miniaci, ${ }^{1,2,3,{ }^{*}}$ R. K. Pal, ${ }^{1}$ B. Morvan, ${ }^{2}$ and M. Ruzzene ${ }^{1}$ \\ ${ }^{1}$ School of Aerospace Engineering and School of Mechanical Engineering, \\ Georgia Institute of Technology, North Avenue, Atlanta, Georgia 30332, USA \\ ${ }^{2}$ University of Le Havre, Laboratoire Ondes et Milieux Complexes, UMR CNRS 6294, \\ 75 Rue Bellot, 76600 Le Havre, France \\ ${ }^{3}$ EMPA, Laboratory of Acoustics and Noise Control, Überlandstrasse 129, 8600 Dübendorf, Switzerland
}

(Received 23 May 2018; revised manuscript received 14 August 2018; published 18 September 2018)

Topologically protected waves in classical media provide unique opportunities for one-way wave transport and immunity to defects. Contrary to acoustics and electromagnetics, their observation in elastic solids has so far been elusive because of the presence of multiple modes and their tendency to hybridize at interfaces. Here, we report on the experimental investigation of topologically protected helical edge modes in elastic plates patterned with an array of triangular holes, along with circular holes that produce an accidental degeneracy of two Dirac cones. Such a degeneracy is subsequently lifted by careful breaking of the symmetry along the thickness direction, which emulates the spin orbital coupling in the quantum spin Hall effect. The joining of two plates that are mirror-symmetric copies of each other about the plate midthickness introduces a nontrivial interface that supports helical edge waves. The experimental observation of these topologically protected wave modes in elastic continuous plates opens avenues for the practical realization of structural components with topologically nontrivial waveguiding properties and their application to elastic waveguiding and confinement.

DOI: 10.1103/PhysRevX.8.031074

\section{INTRODUCTION}

Topological protection provides a significant potential to achieve one-way, defect immune, and scattering free wave propagation [1]. Originally investigated in quantum systems, these concepts have been extended to several classical areas of physics, including acoustic [2-5], electromagnetic [6-8], elastic [9] and optomechanical [10] media.

In mechanical systems, there are two broad ways to achieve topological protection [11]. The first one involves active devices that break time-reversal symmetry and thus mimic the quantum Hall effect [12]. Several theoretical studies and experimental demonstrations have employed microtubules [13], rotationally biased components in ordered structures [14-19], as well as active liquids flowing in square and Lieb lattices [20]. A second approach seeks to establish analogues with the quantum spin Hall effect (QSHE) [21] through solely passive components. Key to

\footnotetext{
* Corresponding author. marco.miniaci@gmail.com

Published by the American Physical Society under the terms of the Creative Commons Attribution 4.0 International license. Further distribution of this work must maintain attribution to the author(s) and the published article's title, journal citation, and DOI.
}

Subject Areas: Mechanics, Metamaterials, Topological Insulators this analogy is the nucleation of a double Dirac cone and the coupling of two degenerate modes corresponding to distinct irreducible representations of the reciprocal lattice symmetry group characterized by a Dirac dispersion [22].

In this context, elastic plates emerge as excellent candidates due to the presence of an infinite number of modes with distinct polarizations and coupled deformation mechanisms. While this is attractive in terms of the quest for topological phases, the high modal density of the wave spectrum in plates [23] makes the opening of an isolated band gap challenging due to the tendency of the various modes to couple and convert at boundaries and interfaces. Indeed, in contrast to the sole presence of a longitudinal wave mode in acoustic media [2], and of transverse modes in electromagnetic media [8,24], a bulk elastic solid supports one longitudinal and two transverse wave modes. Plates are generally defined as solids where one of the dimensions (thickness) is much smaller than the other two. In plates, elastic wave propagation nominally occurs via an infinite number of Lamb modes that are associated with symmetric and antisymmetric deformation profiles across the thickness. These modes are characterized by coupled in-plane and transverse displacements, and they easily hybridize in the presence of interfaces or geometry changes. Geometry and material modifications, in general, affect the dispersion behavior and the associated band 
structure of plates. For example, breaking translation symmetry by introducing periodic holes or inclusions may open band gaps, which, however, may not be complete and may therefore affect only some of the modes. The opening of topologically nontrivial band gaps also requires the structure to maintain certain symmetries (see Sec. II). This further complicates the task of inducing and observing topologically protected wave motion in continuous elastic plates, which so far has been limited to theoretical and numerical studies. The first of such studies is the work of Mousavi et al. [22], where a deep subwavelength patterning in a dual-scale phononic slab is considered. The resulting configuration is of significant complexity and challenging to physically implement. More recently, several studies have exploited valley degrees of freedom to create spinpolarized band gaps and quantum valley Hall effect (QVHE) analogues. This is conveniently achieved by breaking spatial inversion symmetry at the unit cell level, which is an approach that has been successfully applied for flexural waves in plates $[25,26]$ as well as in mechanical lattices with resonators [27].

Inspired by the work in Ref. [22], here we report on a procedure that identifies QSHE analogues for elastic plates of reduced complexity. The approach exploits the sensitivity to macroscopic modifications within the unit cell introduced by through or blind holes, which break the through-the-thickness symmetry and induce a spin-orbitlike effect. The resulting plate configuration is easy to fabricate, and as such, it enables the experimental characterization of a mechanical QSHE analogue in the form of a continuous elastic medium, as opposed to the discrete configuration documented in Ref. [28].

The paper is organized as follows: Section II discusses the approach for achieving a topological band gap that supports localized and isolated interface modes, while Sec. III presents the experimental observation of scattering free wave propagation along sharp corners. Finally, Sec. IV summarizes the main findings of the paper and describes future investigations as well as potential applications of the proposed approach to the identification of QSHE continuous mechanical analogues.

\section{QSHE ANALOGY THROUGH DISPERSION ENGINEERING}

The first step in the process is to consider the patterned plate (PP) with an array of triangular holes as shown in Figs. 1(a) and 1(b). While the plate geometrically resembles the twisted Kagome lattice described in Ref. [29], it is a continuous medium where no lumped elements such as point masses and springs can be identified, as in prior studies on Kagome lattices.

The dynamic response of the plate is governed by the elastic equilibrium equation $\rho \ddot{\boldsymbol{u}}=(\lambda+\mu) \nabla(\nabla \cdot \boldsymbol{u})+$ $\mu \nabla^{2} \boldsymbol{u}=\mathbf{0}$, where $\rho$ is the density of the material, $\boldsymbol{u}$ denotes the displacement vector field, and $\lambda$ and $\mu$ are the Lamé constants. The discretized form of this equation is employed within the COMSOL finite element (FE) environment to compute the dispersion diagrams through the application of Bloch-Floquet theory. To this end, we impose Bloch-periodic conditions on a unit cell along the lattice vectors $\mathbf{a}_{1}$ and $\mathbf{a}_{2}$ [Fig. 1(a)]. The resulting eigenvalue problem $\left(\mathbf{K}-\omega^{2} \mathbf{M}\right) \mathbf{u}=\mathbf{0}$ is solved by varying the wave vector $\mathbf{k}$ along the boundary of the irreducible Brillouin zone $[\Gamma, M, K]$, with $\Gamma \equiv(0,0), M \equiv(0,2 \pi / \sqrt{3})$, and $K \equiv(2 \pi / 3,2 \pi / \sqrt{3})$, which yields the dispersion diagram (band structure) for the bulk. The employed mesh considers four-node tetrahedral elements and is sufficiently refined to provide accurate eigensolutions up to the frequency of interest [30]. Details of geometry, material properties, and the employed FE mesh are provided in Ref. [31].

The plate's band structure exhibits two distinct Dirac points [denoted as D1 and D2 in Fig. 2(a)]. Circular through-the-thickness holes $(\mathrm{TH})$ arranged as in Figs. 1(a) and 1(b) produce an isolated double Dirac point [32], denoted as 2D in Fig. 2(c). Blind holes (BH) through part of the plate thickness break the $\sigma_{h}$ symmetry [28,33] while preserving the original $C_{3 v}$ symmetry of the PP, and they promote the coupling between the modes spanning the Dirac points, thereby emulating the spin orbital interaction in the QSHE $[1,21]$. In contrast to valley modes where the two sets of modes are associated with two distinct valleys $\left(K, K^{\prime}\right)$, here we pursue two distinct sets of modes at each of the high-symmetry points according to the configuration investigated in Ref. [22].

The dispersion curves in Figs. 2(a), 2(c), and 2(e) are color-coded based on a polarization coefficient $p$ that quantifies the mode polarization according to the expression presented in Ref. [31] and originally introduced in Ref. [34]. The polarization factor color bar varies gradually from 0 (blue: predominantly in-plane) to 1 (red: predominantly out-of-plane).

For the PP configuration, both Dirac points reported in Fig. 2(a) correspond to essential degeneracies arising from the plate having $D_{3 h}$ symmetry. An examination of the mode shapes [Fig. 2(b)] reveals that the D1 and D2 modes span the subspaces associated, respectively, with the $E^{\prime}$ and $E^{\prime \prime}$ irreducible representations $[35,36]$ of the reciprocal lattice group of wave vector $D_{3 h}$ at the $K$ point. Furthermore, the mode shapes reveal that D1 and D2 are characterized by dissimilar polarizations. This implies that geometric modifications preserving $D_{3 h}$ symmetry, such as circular holes through the plate thickness [TH in Fig. 1(b)], preserve the Dirac points but change their frequency values. This allows us to selectively shift the curves until the nucleation of a fourfold degeneracy with two overlaid Dirac cones [point 2D in Fig. 2(c)] is obtained. Here, the double Dirac cone is achieved as an accidental degeneracy $[37,38]$ by introducing through holes of radius $r=0.085 a$, where $a$ denotes the magnitude of the lattice vectors [see Fig. 1(a)]. 


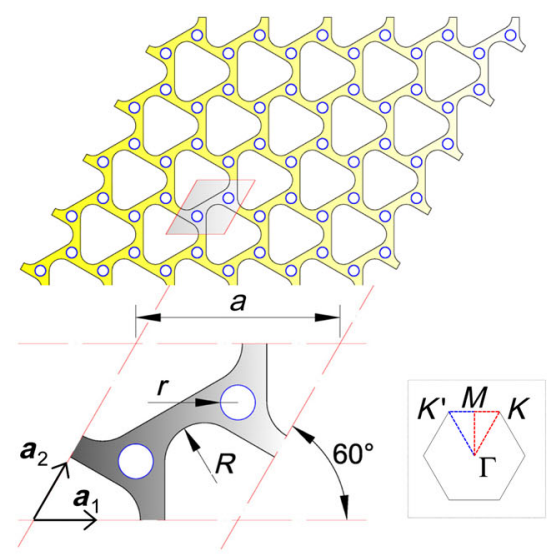

(a)

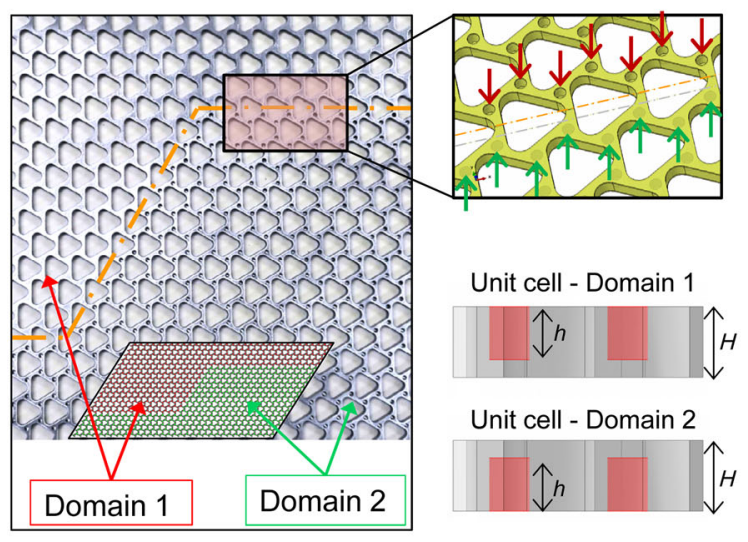

(c)

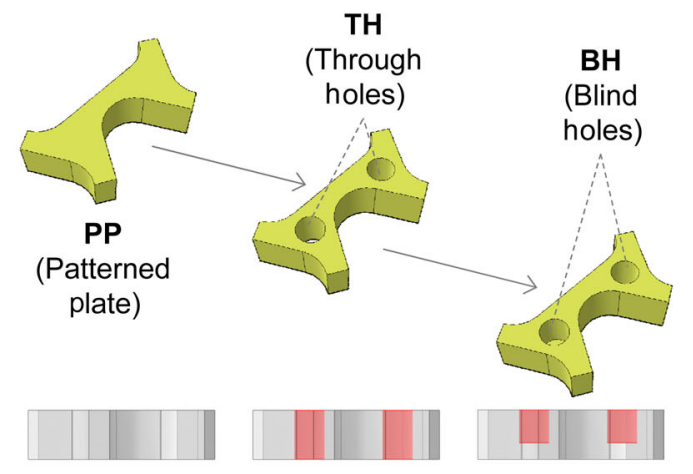

(b)
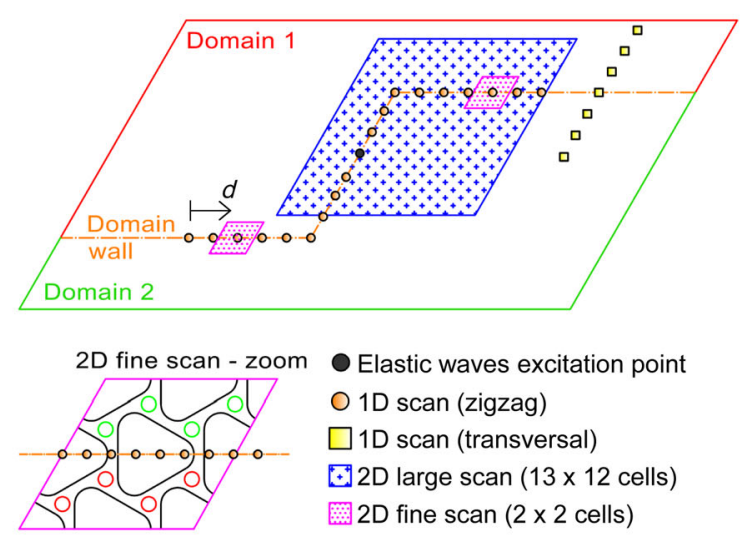

- Elastic waves excitation point

O 1D scan (zigzag)

$\square 1 \mathrm{D}$ scan (transversal)

$[+72 \mathrm{D}$ large scan $(13 \times 12$ cells $)$

周 2D fine scan $(2 \times 2$ cells $)$

(d)

FIG. 1. Patterned plate with a topologically protected interface supporting helical edge modes and the experimental configurations. (a) In-plane view of the patterned plate and schematic representation of the unit cell. The inset shows the $\Gamma, K, K^{\prime}$, and $M$ high-symmetry points of the first irreducible Brillouin zone along which the dispersion curves presented in Figs. 2(a), 2(c), and 2(e) are calculated. (b) Perspective and cross-sectional view of the unit cells used to selectively control dispersion branches: patterned plate (PP), plate with additional circular holes drilled through the thickness (TH) and with blind holes (BH). (c) Photograph of the specimen with a $\mathcal{Z}$-shaped interface (orange dashed line) separating two domains with blind holes drilled on the opposite (top or bottom) surfaces of the plate. The inset shows the arrangement of the reversed unit cells on the full specimen $(20 \times 33$ unit cells) highlighted by the red (domain 1$)$ and green (domain 2) arrows. The top and bottom parts of the right panel show a zoom of the domain wall and the cross sections of the domain-1 and domain-2 unit cells with the reversed holes, respectively. (d) Summary of the experimental scans conducted on the plate for the investigation of its topological waveguiding characteristics. Elastic waves are excited by means of a piezoelectric transducer bonded at the location marked by the circular black dot. The picture illustrates SLDV 1D scans following the $\mathcal{Z}$-shaped interface (orange circular dots) and transverse to the domain wall (yellow square dots), as well as 2 D scans on $13 \times 12$ unit cell (blue) and $2 \times 2$ unit cell (magenta) areas. The letter $d$ indicates the curvilinear coordinate running along the interface.

Breaking the degeneracy at $K$ and coupling the D1 and D2 modes opens a topological band gap [Fig. 2(e)]. Indeed, in contrast to through holes, which preserve the $\sigma_{h}$ symmetry without the occurrence of mode coupling [Fig. 2(d)], blind holes [BH geometry in Fig. 1(b)] couple the predominantly in-plane (D1) and out-of-plane (D2) modes, activating mode hybridization [Fig. 2(f)]. Thus, breaking the $\sigma_{h}$ symmetry of the plate lifts the degeneracy of both the D1 $\left(E^{\prime}\right)$ and D2 $\left(E^{\prime \prime}\right)$ modes. We find that the depth of the BHs plays a key role in both (i) the relative width of the topologically protected band gap and (ii) the shape of the dispersion branches of the edge modes. Figure 3 shows a parametric study on the height $h$ of the BHs. Specifically, the dispersion diagrams for $h / H=0.98$, 0.92 , and 0.58 , where $h$ is the hole depth and $H$ is the plate thickness, are given.

The analysis of Fig. 3 suggests that decreasing the height of the $\mathrm{BH}$ causes the relative width of the topologically protected band gap to initially increase and then to decrease. Specifically, Fig. 3(a) reports the diagram for $h / H=0.98$, i.e., when a very small perturbation with respect to the $\mathrm{TH}$ configuration is introduced and an 


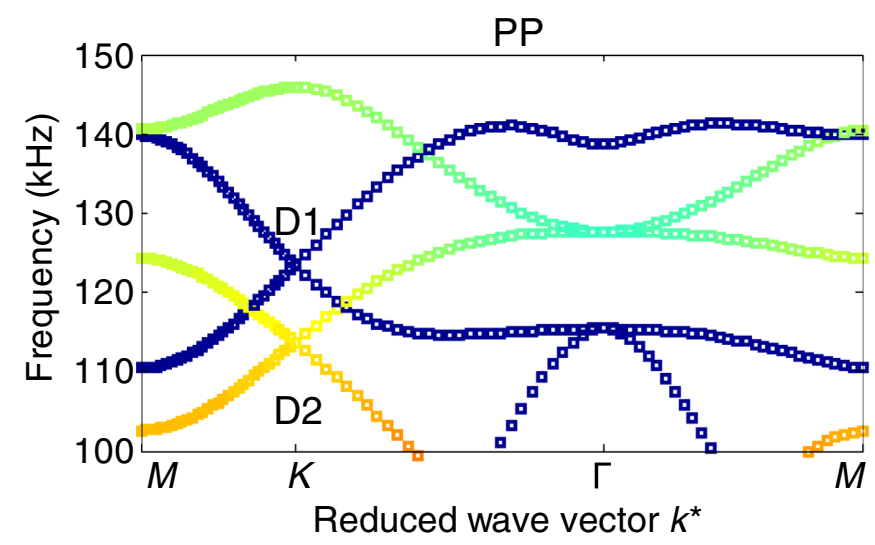

(a)

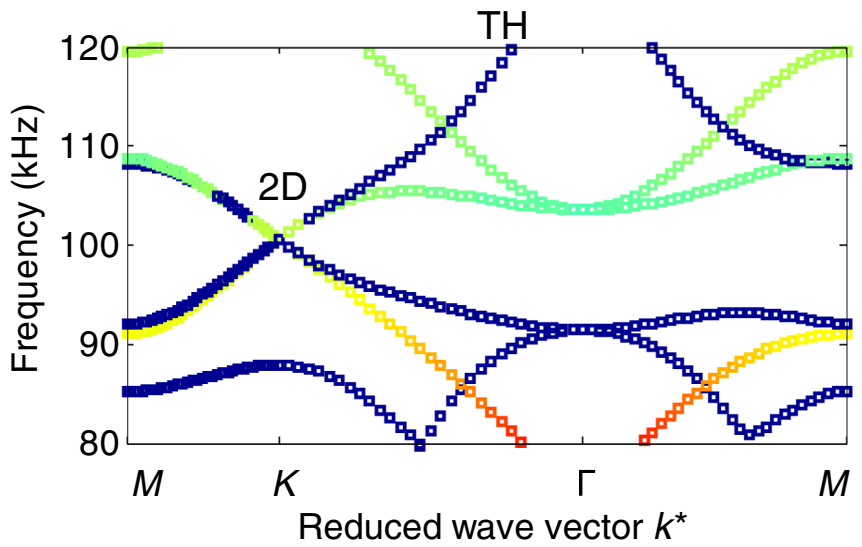

(c)

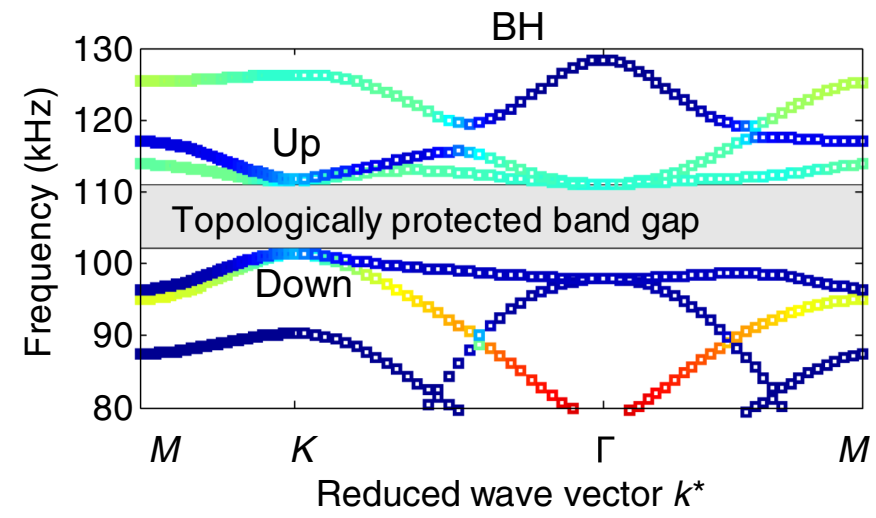

(e)

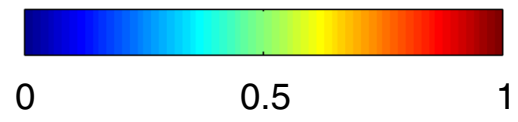

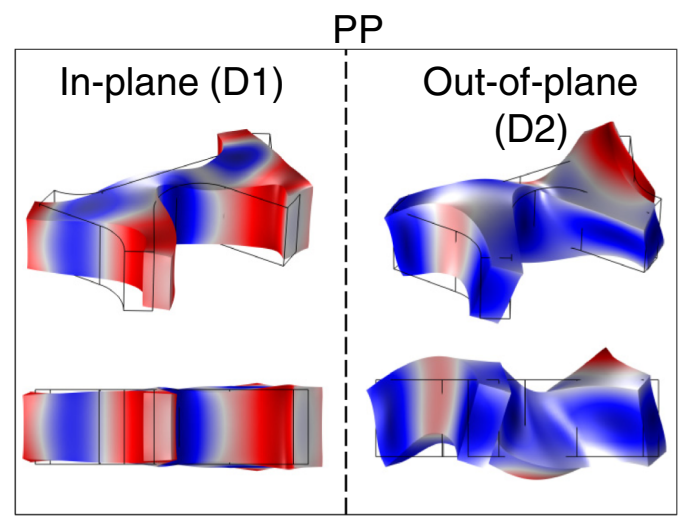

(b)

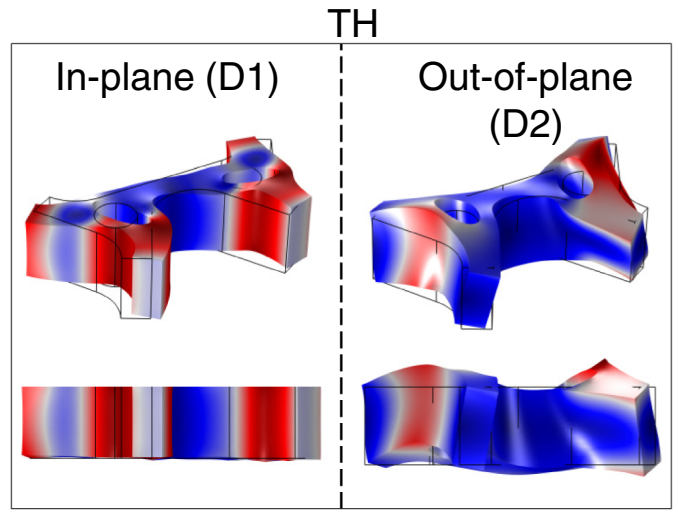

(d)

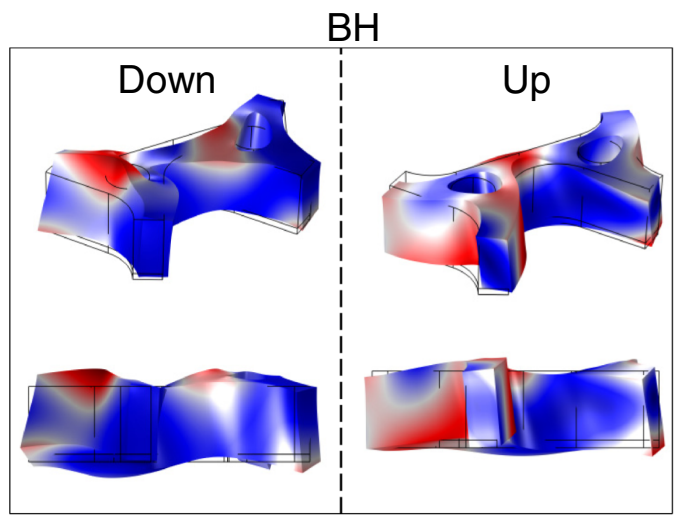

(f)

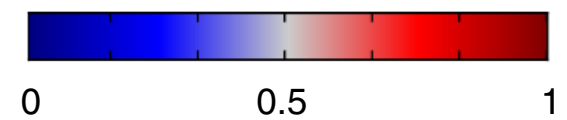

FIG. 2. Selective dispersion curve manipulation for the double Dirac cone nucleation and breaking of the $\sigma_{h}$ mirror symmetry for the opening of a topologically nontrivial band gap. (a) Band structures for the PP exhibiting two separate Dirac points D1 and D2 at the $K$ point. Different mode polarizations are indicated by colors, ranging from pure in-plane (blue) to pure out-of-plane (red) [the same metric applies to Figs. 2(c) and 2(e)]. (b) Wave modes D1 and D2 at the $K$ point for the PP configuration characterized by dissimilar polarizations. The colors denote the normalized displacement [the same metric applies to Figs. 2(d) and 2(f)]. (c) Band structures for the plate with additional TH showing the double degenerate Dirac point 2D. (d) Wave modes D1 and D2 at the $K$ point for the TH configuration, which preserves the $\sigma_{h}$ symmetry without the occurrence of mode coupling. (e) Band structures for the plate with BH characterized by the nucleation of a topological band gap of $9.7 \%$ relative width. (f) Coupling of the D1 and D2 modes responsible for the opening of the topological band gap. 

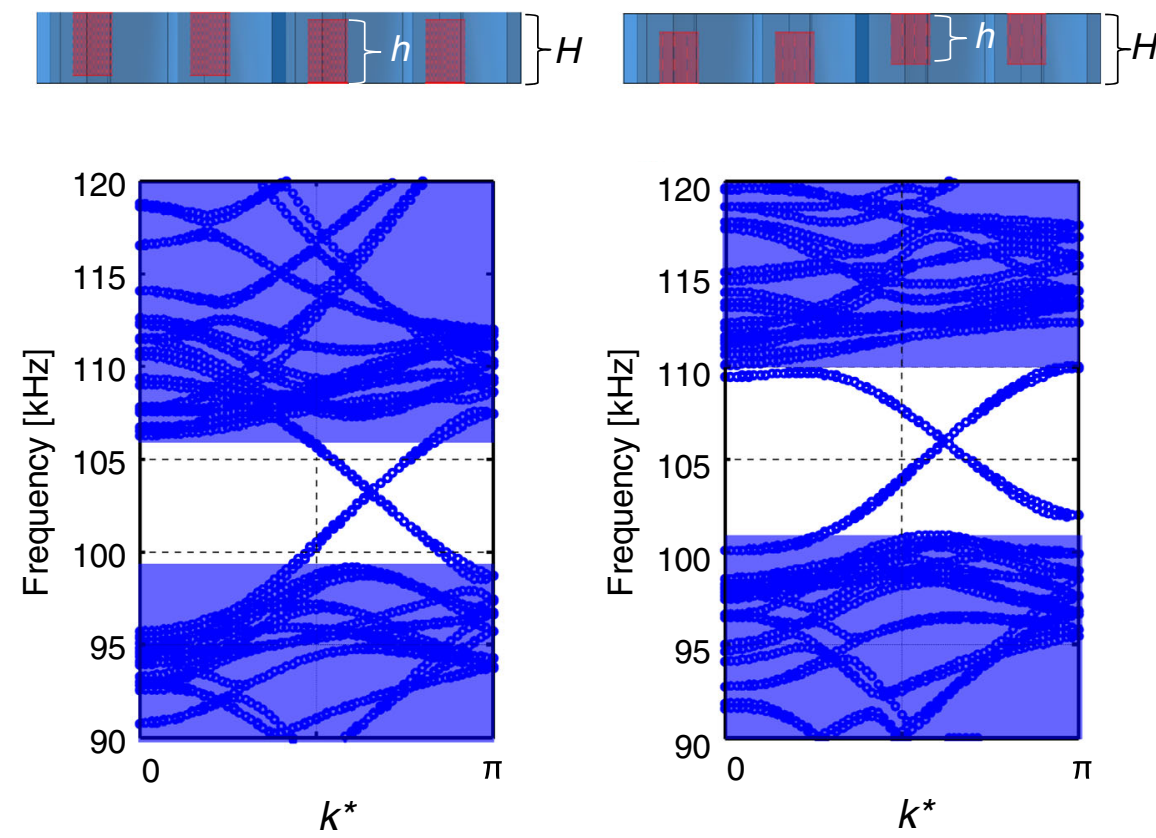

(a)

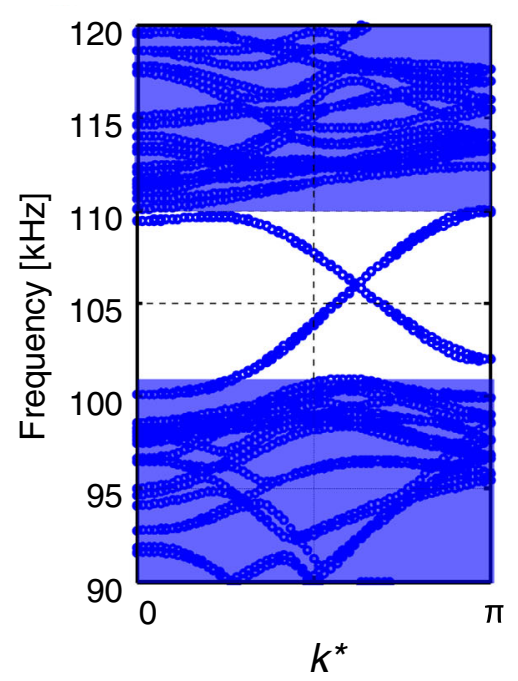

(b)

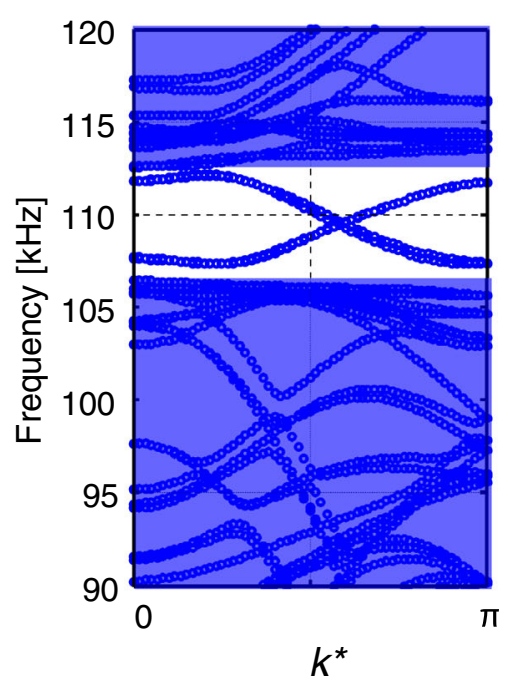

(c)

FIG. 3. Effect of the depth of the blind holes on the dispersion diagrams. (a)-(c) The dispersion diagrams for $10 \times 1$ strips periodic in the $\mathbf{a}_{1}$ direction with a domain wall and blind hole depth of (a) $h / H=0.98(H-h=0.1 \mathrm{~mm})$, (b) $h / H=0.92(H-h=0.5 \mathrm{~mm})$, and (c) $h / H=0.58(H-h=2.5 \mathrm{~mm})$. Decreasing the height of the blind holes causes the relative width of the band gap to initially increase and then to decrease; it also alters the portions of the edge modes exhibiting a linear dispersive behavior.

extremely thin portion of the plate is left pristine $(0.1 \mathrm{~mm})$. In this case, the band gap has a relative width of $6.1 \%$, and the branches of the edge modes connecting the bulk regions (highlighted by the blue rectangles in Fig. 3) exhibit a linear dispersive behavior in almost the entire frequency range of the bulk band gap. Also, for this depth of the blind holes, the interface modes span the entire band gap, in contrast to trivial defect modes, which typically span only part of the band gap. The corresponding two eigenvectors (modes) are, respectively, characterized by clockwise and anticlockwise polarizations, which is illustrated by the phase fields of the mode shapes (see Sec. III). Decreasing the hole depth widens the band gap, for instance, up to $9.3 \%$ in the case of $h / H=0.92$ [Fig. 3(b)]. The branches of the edge modes connecting the bulk regions still exhibit a mainly linear dispersive behavior in almost all the frequency range of the bulk band gap, although some portions have a nonlinear dispersive behavior emerging from the bulk band gap. As the depth of the BH decreases further [Fig. 3(c)], the band gap shrinks. This observation allows us to conclude that the band-gap opening and coupling of modes emulating spinorbit interaction are mainly valid for small perturbations in hole depth. As the perturbation becomes larger, we approach the PP dispersion diagrams, where the two sets of bands separate and are no longer coupled.

In the following investigations, we consider a hole depth corresponding to $h / H=0.9$, which converts the fourfold degeneracy into an approximately $10 \%$-width nontrivial band gap. In addition, the absence of other modes at nearby frequencies guarantees that these hybridized modes remain isolated. Joining two lattices with unit cells related by a $\sigma_{h}$ transformation produces an interface that supports topologically protected helical modes. The existence of such modes is a consequence of the bulk boundary correspondence principle [1], as the hybridized bulk modes on either side are distinct and related by a $\sigma_{h}$ transformation. In contrast, at the free boundary, these localized modes hybridize, become defect modes, and do not span the bulk band gap [22].

\section{EXPERIMENTAL OBSERVATION OF HELICAL EDGE MODES}

To confirm the emergence of topologically protected helical edge modes, we fabricate a plate of $20 \times 33$ unit cells with a $\mathcal{Z}$-shaped interface [dotted orange line in Figs. 1(c) and 1(d)] that separates two domains with reversed blind holes. This path is chosen to illustrate the absence of backscattering in the presence of sharp corners within an interface aligned with the lattice vectors. The numerically predicted dispersion band structure [white curves in Figs. 4(a) and 4(b)], calculated for a $10 \times 1$ strip periodic in the $\mathbf{a}_{1}$ direction, confirms that the system exhibits a pair of helical edge modes, respectively denoted as $M^{-}$and $M^{+}$, where the superscripts - and + indicate the direction of propagation (leftward or rightward) with respect to the excitation point [black dot in Fig. 1(d)]. We remark here that the edge modes do not merge into the bulk 


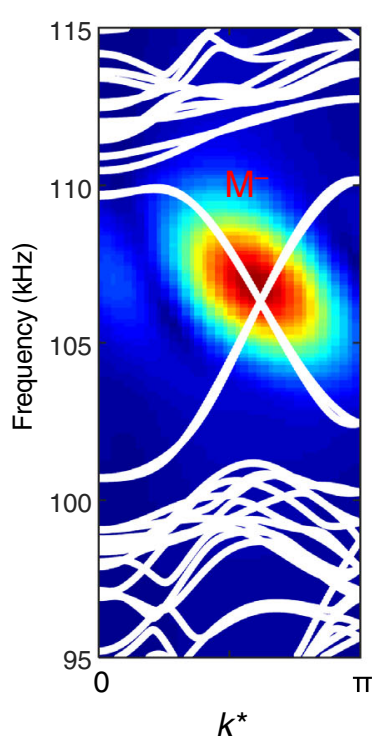

(a)

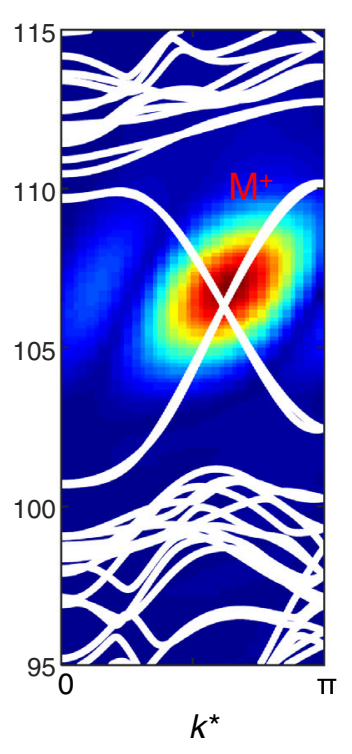

(b)

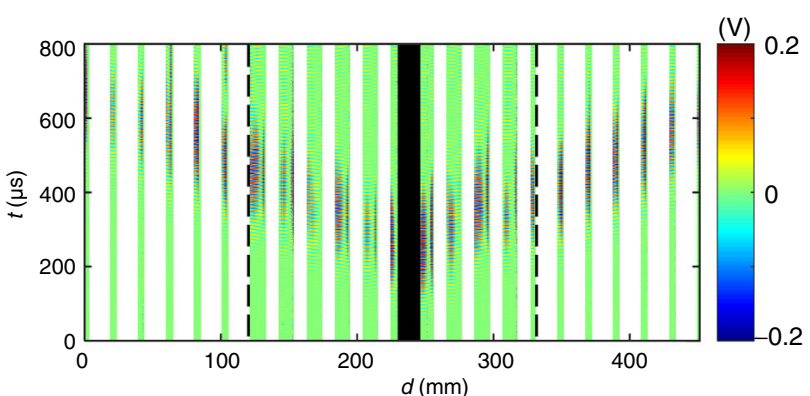

(c)
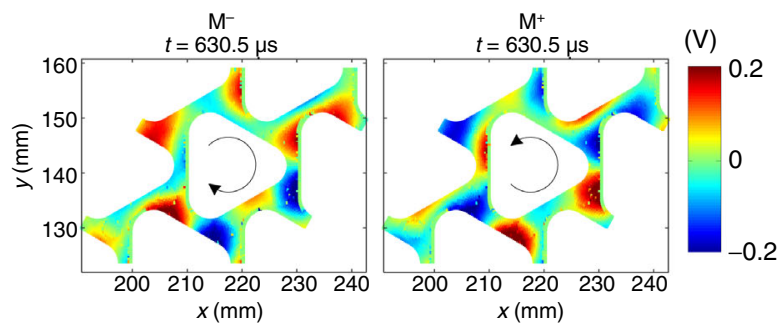

(d)

FIG. 4. Observation of the topologically protected helical edge modes. (a,b) Measured dispersion curves for the left- and rightpropagating edge modes (denoted as $M^{-}$and $M^{+}$, respectively), compared to the numerically predicted band structure (white lines). (c) Spatio-temporal representation of the plate response recorded during the 1D SLDV scan along the $\mathcal{Z}$-shaped interface. The total length of the scanned line is $430 \mathrm{~mm}$ and includes the bottom-straight, oblique, and top-straight segments [orange circular dots in Fig. 1(d)]. The black rectangle positioned at $d=230 \mathrm{~mm}$, where $d$ denotes the curvilinear coordinate along the interface, denotes the position of the piezoelectric transducer used for excitation. White rectangles correspond to holes in the patterned plate where SLDV measurements were not taken. The two dashed black vertical lines mark the location of the turns along the interface. (d) Measured wave field for the left- and right-propagating edge modes $M^{-}$and $M^{+}$displaying typical vortex profiles. The black arrows denote anticlockwise and clockwise vorticity of the displacement field. The measurement corresponds to the area denoted as " $2 \mathrm{D}$ fine scan" in Fig. 1(d). The SLDV sensitivity is set to $20 \mathrm{~mm} / \mathrm{s} / \mathrm{V}$ for all the measurements presented.

spectrum, in contrast to a quantum spin Hall insulator [21] or its exact mechanical analogue [28]. Indeed, as discussed above, the chosen plate design is a true analogue only in the limit of infinitesimal perturbation of the double Dirac cone. However, the chosen design is a good compromise between having a sufficiently wide band gap for the experimental measurements and a small enough perturbation so that the mode spans the full band gap. As demonstrated in the sequence of band diagrams with decreasing blind hole depth in Fig. 3, the edge modes deviate further from the idealized behavior of spanning the full band-gap frequency range. There is a small set of frequencies in the band gap (near the bulk bands), which the edge modes do not span; we consider, based on the results found, that it has a negligible impact on topological protection [Fig. 3(b)].

The existence of the topologically protected modes $M^{-}$ and $\mathrm{M}^{+}$is verified experimentally by measuring the out-ofplane velocity component of the plate surface through a scanning laser Doppler vibrometer (SLDV). A onedimensional (1D) line measurement scan is first conducted along the orange dotted line shown in Fig. 1(d). The measurements record the velocity of points along the line corresponding to the excitation applied by the piezoelectric transducer at the location marked by the solid black circle in Fig. 1(d). The results are presented in the form of a space-time plot, shown in Fig. 4(c), where the spatial coordinate $d$ runs along the orange dotted line within the interface. In Fig. 4(c), the black rectangle denotes the excitation location, while white rectangles correspond to holes in the plate where no measurements were taken. Also, two dashed black vertical lines mark the location of the corners along the interface. The plot illustrates the propagation of the $M^{-}$and $M^{+}$modes that emanate from the excitation location and propagate past the sharp corners with no visible reflections, thus demonstrating the absence of backscattering at the sharp corners. Next, these results are represented in the frequency-wave number domain by performing a temporal and spatial Fourier transform (2D-FT). The magnitude of the resulting 2D-FT is superimposed as a colored surface (with colors varying from blue to red for increasing magnitude values) to the theoretical dispersion predictions for the finite strip in Figs. 4(a) and 4(b). This representation, which maps the wave response and effectively separates the modes in the frequency-wave number domain, further confirms the existence of the pair of edge modes inside the bulk band gap and shows an excellent match with their numerical prediction. 

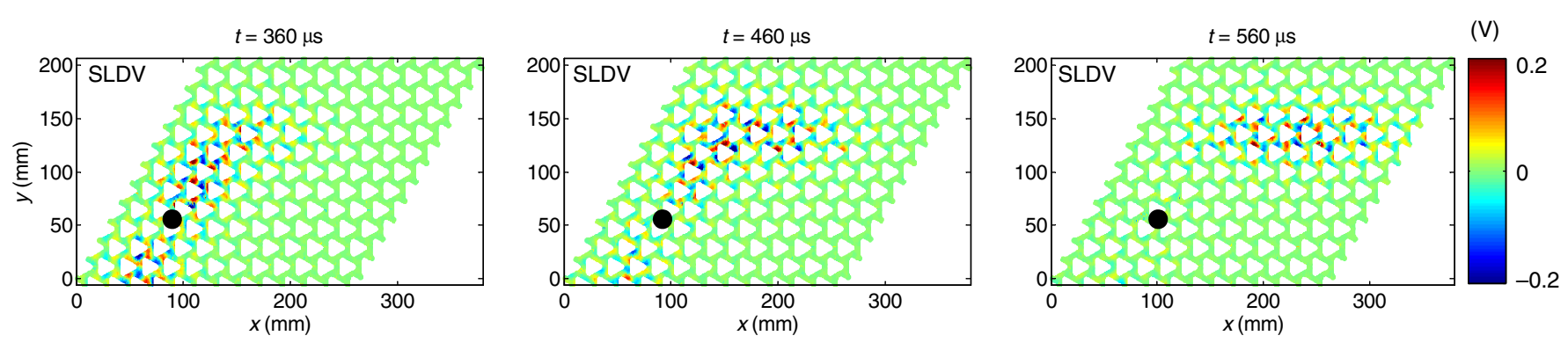

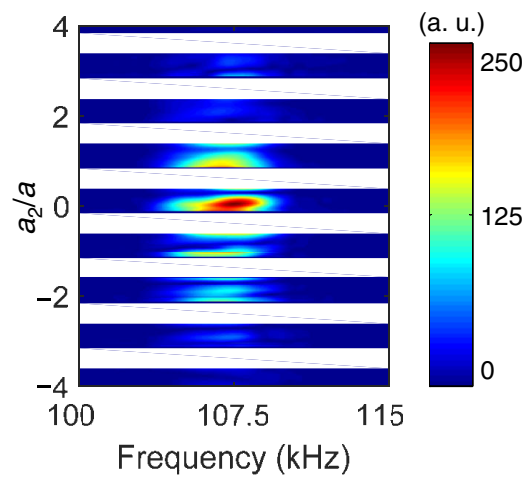

(b)

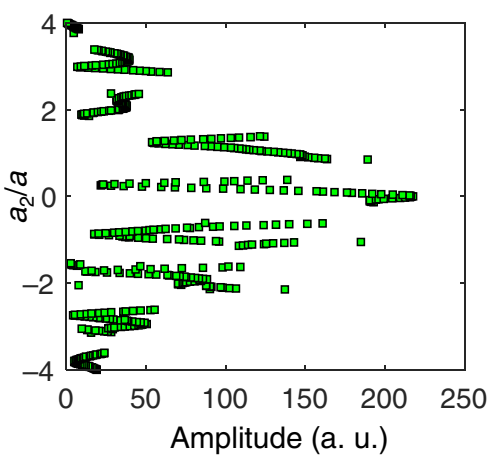

(c)

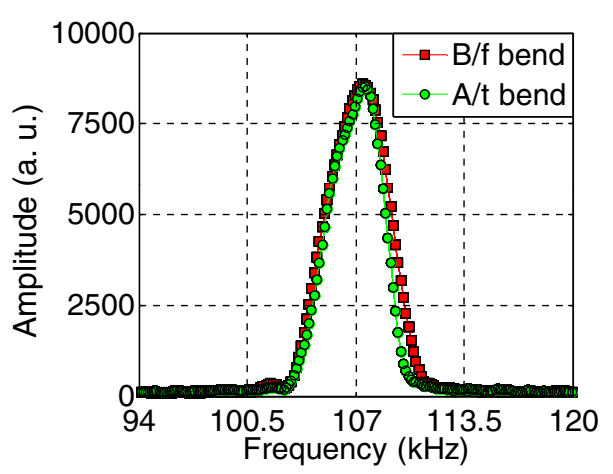

(d)

FIG. 5. Immunity to backscattering of the helical edge waves along the $\mathcal{Z}$-shaped interface. (a) Experimental wave-field measurements showing the right-propagating edge mode traversing the turn in the top-right portion of the plate [the domain of measurements is denoted as "2D large scan" in Fig. 1(d)]. The time snapshots show the out-of-plane velocity before the wave approaches the turn (at $t=360 \mu$ s) and demonstrate how the nontrivial interface supports the propagation past the $120^{\circ}$ bend without backscattering $(t=460 \mu \mathrm{s})$ while sustaining propagation after the $\mathcal{Z}$ bend $(t=560 \mu \mathrm{s})$. The colors, varying from blue to red, correspond to the out-of-plane velocity of the plate (1 V corresponds to a velocity of $20 \mathrm{~mm} / \mathrm{s}$ ). (b) Experimental amplitude profile across the interface for the right-propagating edge mode as a function of the frequency. Signals are acquired along a line in the $\mathbf{a}_{2}$ direction, transverse to the domain wall in the top part of the waveguide [see yellow square dots in Fig. 1(d)]. (c) Spectral amplitude at $f=107 \mathrm{kHz}$ as a function of the distance from the interface. (d) Comparison of amplitudes of the 1D-FT averaged over 1D scan lines of the same length located before and after the upper sharp bend of the waveguide, quantitatively illustrating the absence of backscattering or mode conversion after the $120^{\circ}$ bend. The SLDV sensitivity is set to $20 \mathrm{~mm} / \mathrm{s} / \mathrm{V}$ for all the measurements presented.

Next, the polarization characteristics of the $M^{-}$and $M^{+}$ modes are investigated through a local scan conducted over the $2 \times 2$ unit cell areas shown in violet in Fig. 1(d). Snapshots of the recorded wave-field velocity distributions for the two modes at representative time instants are presented in Fig. 4(d). Videos provided in Ref. [31] are instructive in describing the different motion patterns of the modes. Specifically, the two modes are characterized, respectively, by a clockwise and an anticlockwise vorticity of the velocity field with respect to the interface, which can clearly be appreciated from the analysis of the videos of Ref. [31]. Here, such vorticity is highlighted by black arrows drawn on the basis of the phase of the measured wave field. This vortex behavior may be viewed as a pseudospin analogous to the $A-B$ sublattice or the topbottom layer pseudospin in graphene systems [39].

The interface bound propagation and the absence of significant backscattering at a sharp bend $\left(120^{\circ}\right)$ are illustrated by the SLDV wave-field measurements over the region labeled as "2D large scan" in Fig. 1(d).
Figure 5(a) shows the measured out-of-plane velocity distribution resulting from a sine burst excitation applied at the location of the black dot. The frequency content of the input signal (51 sine cycles modulated by a Hanning window of central frequency $107 \mathrm{kHz}$ ) is chosen so that the excitation bandwidth falls entirely inside the bulk band gap, in an attempt to prevent bulk mode excitation. Snapshots of the recorded wave field - at instants before, during, and after the edge mode has entered the turn-show how a change in the interface direction does not affect the propagating nature of the wave and does not lead to backscattering, which would be expected in the case of a trivial interface. In addition, weak penetration inside the bulk region is observed. Keeping the excitation frequency content intentionally centered inside the bulk band gap minimizes the excitation of bulk modes and allows us to quantitatively estimate the decay of the field amplitude away from the interface, which is evaluated here by conducting an additional 1D measurement scan along a line transverse to the domain wall [denoted by the yellow square dots in Fig. 1(d)]. 
The acquired signals are postprocessed by computing the Fourier transform in time (1D-FT) at each acquisition point. The magnitude of the resulting 1D-FT as a function of frequency and position along the scan line, normalized here with respect to the lattice constant $a$, is shown in Fig. 5(b), while Fig. 5(c) shows the amplitude variation as a function of transverse location at the specific frequency of $107 \mathrm{kHz}$. Both figures help us to visualize the rapid spatial decay of the wave away from the interface. Figure 5(d) compares the amplitudes of 1D-FTs averaged along 1D scan lines of the same length placed before and after the upper sharp bend of the waveguide. The constant spectral content before and after the turn observed in this comparison clearly illustrates the absence of backscattering or mode conversion after the $120^{\circ}$ turn. The change in direction of the wave propagation (before and after the corner) is not accompanied by significant energy losses, thus providing evidence that no mode conversion or backscattering occurs at the turn.

\section{CONCLUSIONS}

The results presented in this work provide the experimental demonstration of the generation of a topological interface, which is robust to backscattering and has the ability to guide waves around sharp corners. The proposed design and the investigations presented herein, both numerical and experimental, provide insights into the behavior of topologically protected helical edge modes in continuous elastic systems. The results suggest a simple process, which may be exploited to explore new avenues [40] for topologically protected wave motion in fields where vibrations play a crucial role, such as civil engineering and the aerospace industry, or where the transmission of information through elastic waves may be an attractive option.

\section{ACKNOWLEDGMENTS}

M. M. has received funding from the European Union's Horizon 2020 research and innovation program under the Marie Skłodowska-Curie Grant Agreement No. 754364. M. M. is also grateful to Dr. M. Mazzotti and Dr. V. Pagneux for insightful discussions. R. K. P. and M. R. acknowledge the support of the EFRI Grant No. 1741685 from the National Science Foundation.

[1] M.Z. Hasan and C.L. Kane, Colloquium: Topological Insulators, Rev. Mod. Phys. 82, 3045 (2010).

[2] Z. Yang, F. Gao, X. Shi, X. Lin, Z. Gao, Y. Chong, and B. Zhang, Topological Acoustics, Phys. Rev. Lett. 114, 114301 (2015).

[3] C. He, X. Ni, H. Ge, X.-C. Sun, Y.-B. Chen, M.-H. Lu, X.-P. Liu, and Y.-F. Chen, Acoustic Topological Insulator and Robust One-Way Sound Transport, Nat. Phys. 12, 1124 (2016).
[4] J. Lu, C. Qiu, L. Ye, X. Fan, M. Ke, F. Zhang, and Z. Liu, Observation of Topological Valley Transport of Sound in Sonic Crystals, Nat. Phys. 13, 369 (2017).

[5] R. Fleury, A. B. Khanikaev, and A. Alu, Floquet Topological Insulators for Sound, Nat. Commun. 7, 11744 (2016).

[6] A. B. Khanikaev, S. H. Mousavi, W.-K. Tse, M. Kargarian, A. H. MacDonald, and G. Shvets, Photonic Topological Insulators, Nat. Mater. 12, 233 (2013).

[7] X. Cheng, C. Jouvaud, X. Ni, S. H. Mousavi, A. Z. Genack, and A. B. Khanikaev, Robust Reconfigurable Electromagnetic Pathways within a Photonic Topological Insulator, Nat. Mater. 15, 542 (2016).

[8] L. Lu, J. D. Joannopoulos, and M. Soljačić, Topological Photonics, Nat. Photonics 8, 821 (2014).

[9] S. D. Huber, Topological Mechanics, Nat. Phys. 12, 621 (2016).

[10] V. Peano, C. Brendel, M. Schmidt, and F. Marquardt, Topological Phases of Sound and Light, Phys. Rev. X 5, 031011 (2015).

[11] R. Süsstrunk and S. D. Huber, Classification of Topological Phonons in Linear Mechanical Metamaterials, Proc. Natl. Acad. Sci. U.S.A. 113, E4767 (2016).

[12] F. D. M. Haldane, Model for a Quantum Hall Effect without Landau Levels: Condensed-Matter Realization of the “Parity Anomaly”, Phys. Rev. Lett. 61, 2015 (1988).

[13] E. Prodan and C. Prodan, Topological Phonon Modes and Their Role in Dynamic Instability of Microtubules, Phys. Rev. Lett. 103, 248101 (2009).

[14] R. Fleury, D. L. Sounas, C. F. Sieck, M. R. Haberman, and A. Alù, Sound Isolation and Giant Linear Nonreciprocity in a Compact Acoustic Circulator, Science 343, 516 (2014).

[15] A. B. Khanikaev, R. Fleury, S. H. Mousavi, and A. Alù, Topologically Robust Sound Propagation in an AngularMomentum-Biased Graphene-like Resonator Lattice, Nat. Commun. 6, 8260 (2015).

[16] P. Wang, L. Lu, and K. Bertoldi, Topological Phononic Crystals with One-Way Elastic Edge Waves, Phys. Rev. Lett. 115, 104302 (2015).

[17] N. Swinteck, S. Matsuo, K. Runge, J. Vasseur, P. Lucas, and P. A. Deymier, Bulk Elastic Waves with Unidirectional Backscattering-Immune Topological States in a TimeDependent Superlattice, J. Appl. Phys. 118, 063103 (2015).

[18] L. M. Nash, D. Kleckner, A. Read, V. Vitelli, A. M. Turner, and W. T. Irvine, Topological Mechanics of Gyroscopic Metamaterials, Proc. Natl. Acad. Sci. U.S.A. 112, 14495 (2015).

[19] G. Salerno, T. Ozawa, H. M. Price, and I. Carusotto, Floquet Topological System Based on Frequency-Modulated Classical Coupled Harmonic Oscillators, Phys. Rev. B 93, 085105 (2016).

[20] A. Souslov, B. C. van Zuiden, D. Bartolo, and V. Vitelli, Topological Sound in Active-Liquid Metamaterials, Nat. Phys. 13, 1091 (2017).

[21] C. L. Kane and E. J. Mele, Quantum Spin Hall Effect in Graphene, Phys. Rev. Lett. 95, 226801 (2005).

[22] S. H. Mousavi, A. B. Khanikaev, and Z. Wang, Topologically Protected Elastic Waves in Phononic Metamaterials, Nat. Commun. 6, 8682 (2015). 
[23] K. F. Graff, Wave Motion in Elastic Solids (Dover Publications, New York, 1991).

[24] T. Ozawa, H. M. Price, A. Amo, N. Goldman, M. Hafezi, L. Lu, M. Rechtsman, D. Schuster, J. Simon, O. Zilberberg, and I. Carusotto, Topological Photonics, arXiv:1802.04173.

[25] R. K. Pal and M. Ruzzene, Edge Waves in Plates with Resonators: An Elastic Analogue of the Quantum Valley Hall Effect, New J. Phys. 19, 025001 (2017).

[26] J. Vila, R. K. Pal, and M. Ruzzene, Observation of Topological Valley Modes in an Elastic Hexagonal Lattice, Phys. Rev. B 96, 134307 (2017).

[27] H. Zhu, T.-W. Liu, and F. Semperlotti, Design and Experimental Observation of Valley-Hall Edge States in Diatomic-Graphene-like Elastic Waveguides, Phys. Rev. B 97, 174301 (2018).

[28] R. Süsstrunk and S. D. Huber, Observation of Phononic Helical Edge States in a Mechanical Topological Insulator, Science 349, 47 (2015).

[29] T. C. Lubensky, C. L. Kane, X. Mao, A. Souslov, and K. Sun, Phonons and Elasticity in Critically Coordinated Lattices, Rep. Prog. Phys. 78, 073901 (2015).

[30] M. Miniaci, A. Marzani, N. Testoni, and L. De Marchi, Complete Band Gaps in a Polyvinyl Chloride (PVC) Phononic Plate with Cross-like Holes: Numerical Design and Experimental Verification, Ultrasonics 56, 251 (2015).

[31] See Supplemental Material at http://link.aps.org/ supplemental/10.1103/PhysRevX.8.031074 for details about the tested specimen, the experimental setup and the data processing, the numerical procedures and the explanation to the movie files.
[32] J. Lu, C. Qiu, S. Xu, Y. Ye, M. Ke, and Z. Liu, Dirac Cones in Two-Dimensional Artificial Crystals for Classical Waves, Phys. Rev. B 89, 134302 (2014).

[33] R. K. Pal, M. Schaeffer, and M. Ruzzene, Helical Edge States and Topological Phase Transitions in Phononic Systems Using Bi-layered Lattices, J. Appl. Phys. 119, 084305 (2016).

[34] M. Miniaci, A. Gliozzi, B. Morvan, A. Krushynska, F. Bosia, M. Scalerandi, and N. Pugno, Proof of Concept for an Ultrasensitive Technique to Detect and Localize Sources of Elastic Nonlinearity Using Phononic Crystals, Phys. Rev. Lett. 118, 214301 (2017).

[35] M. S. Dresselhaus, G. Dresselhaus, and A. Jorio, Group Theory: Application to the Physics of Condensed Matter (Springer Science \& Business Media, New York, 2007).

[36] K. Sakoda, Double Dirac Cones in Triangular-Lattice Metamaterials, Opt. Express 20, 9925 (2012).

[37] X. Huang, Y. Lai, Z. H. Hang, H. Zheng, and C. Chan, Dirac Cones Induced by Accidental Degeneracy in Photonic Crystals and Zero-Refractive-Index Materials, Nat. Mater. 10, 582 (2011).

[38] Z.-G. Chen, X. Ni, Y. Wu, C. He, X.-C. Sun, L.-Y. Zheng, M.-H. Lu, and Y.-F. Chen, Accidental Degeneracy of Double Dirac Cones in a Phononic Crystal, Sci. Rep. 4, 4613 (2014).

[39] D. Xiao, W. Yao, and Q. Niu, Valley-Contrasting Physics in Graphene: Magnetic Moment and Topological Transport, Phys. Rev. Lett. 99, 236809 (2007).

[40] G. Ma and P. Sheng, Acoustic Metamaterials: From Local Resonances to Broad Horizons, Sci. Adv. 2, e1501595 (2016). 